\title{
Simulation studies of atomic resolution X-ray holography
}

\author{
YOGESH KASHYAP, P S SARKAR, AMAR SINHA* and B K GODWAL \\ High Pressure Physics Division, Bhabha Atomic Research Centre, Mumbai 400 085, India
}

MS received 1 September 2003; revised 17 November 2003

\begin{abstract}
X-ray holography is a new method of structure determination based on measurement of interference of a known reference wave with an unknown object wave (containing information on atomic sites scattering the reference wave) so that phase information is preserved. Unlike $X$-ray diffraction, it does not demand for translational periodicity in the material. It is based on the idea similar to that of optical holography and has been tested on crystals, quasicrystals, thin films and doped semiconductors for their structure determination. In order to analyse potentials and limitations of this technique, we have carried out theoretical simulation studies on simple structures. In this paper we describe the basic algorithm of hologram generation and reconstruction of atomic positions from generated data. We illustrate this technique using Fe (bcc) single crystal as sample case to demonstrate its capabilities and limitations. Simulations were carried out on the $\mathrm{Cu}$ ( fcc) structure and on complex structure such as the Al-Pd-Mn quasicrystal. Technical issues such as low signal to noise ratio, twin image problem etc have been discussed briefly to emphasize the need for high intensity $\mathrm{X}$-ray source such as synchrotron for experiments and proper reconstruction algorithm. Finally the scope and potential of this technique have been discussed.
\end{abstract}

Keywords. Simulation studies; back reconstruction studies; X-ray holography.

\section{Introduction}

The need of structure determination is of fundamental importance in understanding and predicting many of the properties of the materials. Routinely used scattering methods (X-ray and electron diffraction) not only demand for a long range periodicity but even the single crystal diffraction methods may have complications due to the phase problem. Recently a new technique, called atomic resolution holography (Gog et al 1996; Tegze and Faigel 1996), has emerged as a new tool in determining the 3D arrangement of atoms inside the bulk of the solid. It demands for only rotational order in the material under investigation. The requirement of long-range periodicity and the need of a priori modeling of the structure is also avoided with the added merit that the phase problem of crystallography finds its natural solution. Moreover, with the advent of the powerful X-ray sources like synchrotrons, one can go for imaging only the environment of selected types of the atoms inside the bulk of the solid. There are many variants of holography like $\gamma$-ray holography, electron holography, neutron holography etc but we shall confine our discussion mostly to X-ray holography. The basic principles of all the variants being the same and they differ only in the experimental realization of the different techniques.

\footnotetext{
*Author for correspondence
}

\section{Basic principle of X-ray holography}

The idea of holography was invented by Gabor (1948) and was extended by Szoke (1986) for obtaining the structural information at atomic level. According to this idea a primary wave, $\mathbf{R}(\mathbf{k})$, called reference wave is mixed with the scattered wave called object wave, $\mathbf{O}(\mathbf{k})$, and the resulting pattern is recorded. The reference and the object wave have to be coherent. The intensity recorded in this process is given by

$$
\begin{aligned}
\mathbf{I}(\mathbf{k}) & =|\mathbf{R}(\mathbf{k})+\mathbf{O}(\mathbf{k})|^{2} \\
& =|\mathbf{R}(\mathbf{k})|^{2}+|\mathbf{O}(\mathbf{k})|^{2}+\mathbf{O}(\mathbf{k}) \mathbf{R}(\mathbf{k})^{*}+\mathbf{R}(\mathbf{k}) \mathbf{O}(\mathbf{k})^{*} .
\end{aligned}
$$

The first two terms are intensities of the reference and object waves, respectively whereas the last two terms correspond to the interference term. Thus both the intensity as well as the phase are recorded. The first interference term is responsible for actual image while second one gives rise to virtual image, called as the twin-image of the first one. Szoke (1986) suggested that one could use the atoms of the solid as the source of radiation. In this way one can obtain atomic resolution limited by the size of the atom and wavelength only. This is called normal $\mathrm{XFH}$ or inside the source concept. This idea relies on the fact that the radiation of the atom has coherence volume large enough to contain the neighbours of the source atom in a cluster or the solid. This emitted radiation can reach the detector in the far field directly or after getting 
scattered from the neighbouring atoms. The direct wave is called reference wave while the scattered wave is called object wave. These two waves then interfere with each other and the detector on the surface records this angle dependent intensity pattern called hologram. This can then be used to reconstruct the 3D images of the environment of the source atom. For a cluster containing large number of atoms, one can have large number of emitters. In that case the hologram will be simply the incoherent superposition of the holograms generated by each emitter. So for the faithful reconstruction, one should have a cluster of atoms having identical rotational order. If this condition was violated then we would get only the average atomic arrangement of the atoms. It may be noted that in X-ray holography methods, the concept of unit cell is not required.

We know from the optical reciprocity principle that if we exchange the detector with the source, then we obtain the same experiment. We now have a source in the far field producing a plane wave impinging on the sample. This plane wave is scattered by the neighbouring atoms and produces an interference pattern inside the sample. If atom can be excited by this radiation, it will have a higher or lower probability of getting excited depending upon the interference between the plane wave and the scattered wave. Now the fluorescence yield due to the atom will be proportional to the field intensity inside the sample. This measurement would give exactly same information, as the normal XFH except the wavelength used in this experiment will be that of the source in the far field. The fluorescence yield is detected as the function of the direction and the wavelength. This is called inverse XFH or internal detector scheme. The hologram so generated is about three orders of magnitude smaller relative to the background. Extraction of hologram from the collected data is therefore a difficult task. So the very first step in the application of this technique is to have very good quality data. Hence all the experiments have so far been done only at synchrotron sources because of their high flux. In the absence of any such experimental data one can generate the same by computer simulation. Here we describe some simulation studies of XFH and inverse XFH on iron $(b c c)$ and copper $(f c c)$ single crystals along with some studies on $i-\mathrm{Al}-\mathrm{Pd}-\mathrm{Mn}$ quasicrystal. The purpose of these simulations is to test the existing reconstruction algorithms and to plan suitable experiments, for example, to verify the predictions of recently proposed cluster-disordered glue model (Modak et al 2000; Chidambaram 2002). Of particular interest in this regard is the distribution of atoms in the glue region in the $\mathrm{Al}-\mathrm{Mn}-\mathrm{Si}$ quasicrystal and its $1 / 1$ crystalline approximants and compare the degree of disorder between them. Note that the inverse XFH (Gog et al 1996), which is predicted to access the buried interfaces and dilute dopants, are expected to address these details.

\section{Simulation of the hologram and back reconstruction studies}

We have carried out some simulation studies of X-ray holography. In this section we will first discuss the method of hologram generation theoretically using the tabulated data and then the reconstruction techniques will be explained with different examples. We report some of our work on iron $(b c c)$ and copper $(f c c)$ single crystals and some preliminary simulation studies on the complex structure such as Al-Pd-Mn alloy.

\subsection{Numerical hologram generation}

The holograms have been generated using the following mathematical expression

$$
\left.\chi(\mathbf{k})=\mid\left(1+r_{\mathrm{e}} \sum F\left(\theta^{k} r_{\mathrm{j}}\right) \exp i(\mathbf{k} \cdot \mathbf{r}-k r)\right) / r_{\mathrm{j}}\right)\left.\right|^{2},
$$

where $r_{\mathrm{e}}$ is the classical electronic radius, $\mathbf{k}$ the wave vector and $F\left(\theta^{k} r_{\mathrm{j}}\right)$ the scattering factor of the atoms assuming them to be the point scatter. This formulation has been derived based on the ideas of classical electrodynamics and quantum corrections do not make any significant contribution to our results and hence have been ignored in our present calculations. We treat $\mathrm{XFH}$ and inverse XFH on the same footing except for the fact that the central atom will be treated as the source in XFH and as the detector in case of inverse XFH. We implement this formulation on a single crystal, iron $(b c c)$, to illustrate the basic idea of the hologram generation and reconstruction. Hologram was generated on the angular range of $\theta=0^{\circ}-90^{\circ}$ and $\varphi=0^{\circ}-360^{\circ}$ for an iron $(b c c)$ cluster having 36 atoms arranged in $b c c$ lattice by subtracting constant background from the obtained intensity distribution. Figure 1 shows the top view of a typical hologram generated using the above formalism at $k=8 \mathrm{~A}^{-1}$. The $\varphi$ rotation axis was parallel to the [001] direction (taken as $z$-direction for simulation purpose) of the $b c c$ iron cluster. The hologram shows the four-fold symmetry along this direction, which corresponds to the four-fold symmetry of $\mathrm{Fe}(b c c)$. As is evident from the above figure the hologram contains valuable symmetry information about the structure and hence one should choose the orientation axis along the direction of the maximum symmetry of the crystal. Moreover, since we are dealing with only small size clusters the problem of the Kossel lines or X-ray standing wave pattern also does not arise. However, in actual experiments done with large size clusters these holographic oscillations may be completely masked out by Kossel lines as can be easily seen from following simulation (figure 2). In order to retrieve the holographic oscillations one has to then apply the low pass filter. This operation also introduces some systematic artifacts in the reconstructed data. 


\subsection{Numerical reconstruction}

Having obtained the hologram either theoretically or experimentally the next step is to reconstruct back the real space atomic positions. This is achieved by the HelmholtzKirchhoff integral theorem (Barton 1988). In the special case when the hologram is obtained on the spherical surface this simplifies to the following:

$$
U(r)=\iint_{s} \chi(\mathbf{k}) \exp i(-\mathbf{k} \cdot \mathbf{r}) \mathrm{d} s,
$$

where $\mathrm{d} s$ is the surface on which the hologram data has been generated.

Figure 3 shows the $2 D$ images of $\mathrm{Fe}(b c c)$ through (001) plane (through the emitter) reconstructed back using the

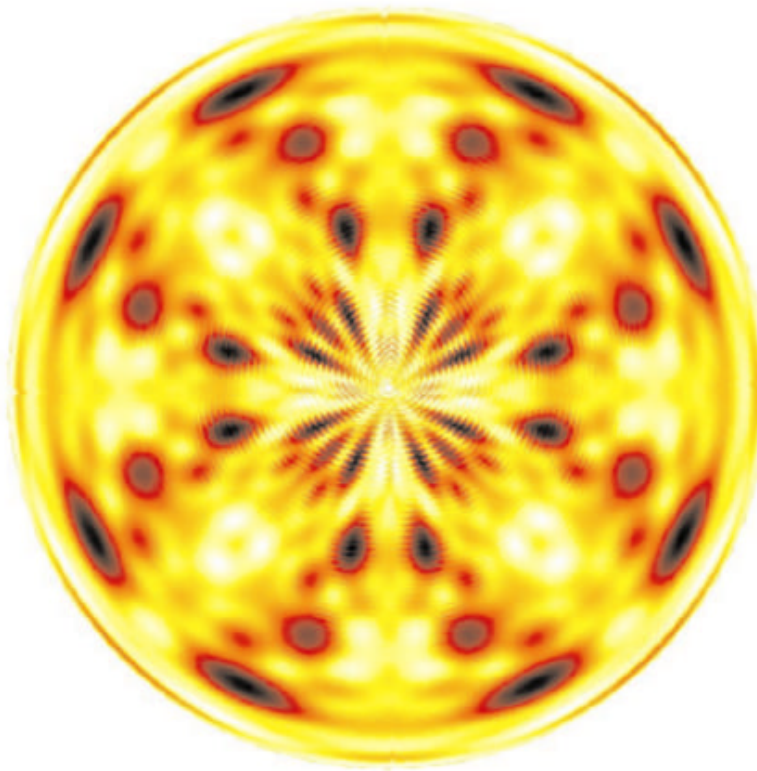

Figure 1. A typical hologram of $\mathrm{Fe}(b c c)$ single crystal at $8^{\circ} \mathrm{A}^{-1}$.

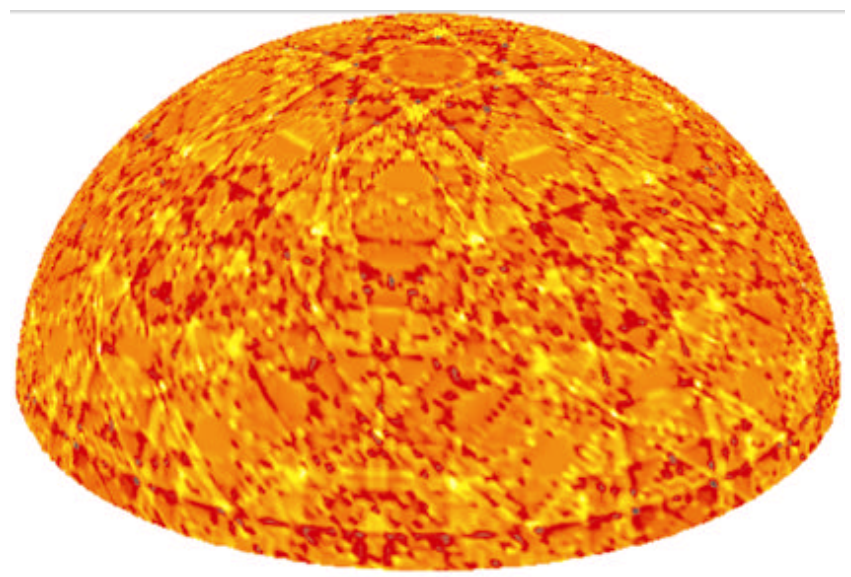

Figure 2. A typical X-ray standing wave pattern generated for a $f \mathrm{cc}$ crystal for illustration purpose. single energy reconstruction and we have plotted $|U(r)|^{2}$ (Kashyap et al 2003).

As it is seen approximately all the atomic positions have been properly reconstructed back yielding the real space configuration of Fe (bcc) cluster. The artifacts present in the reconstruction arise from various approximations that have been used in the simulations. Moreover, since the holograms have been generated only in the limited range and not on the full sphere as demanded by the reconstruction algorithm, the resolution is not expected to be isotropic in all directions as can be seen in figure 4 , where all atomic positions have got elongated. This limited sampling introduces some artifacts in reconstruction. We have also simulated a cluster of copper atoms arranged on the $f c c$ lattice with the above algorithm using $k=8 \mathrm{~A}^{-1}$ for test purpose. A 2D reconstruction passing through (001) plane is shown in the figure. All the atomic positions have been clearly reproduced, however, a large number of artifacts are also present (figure 5).
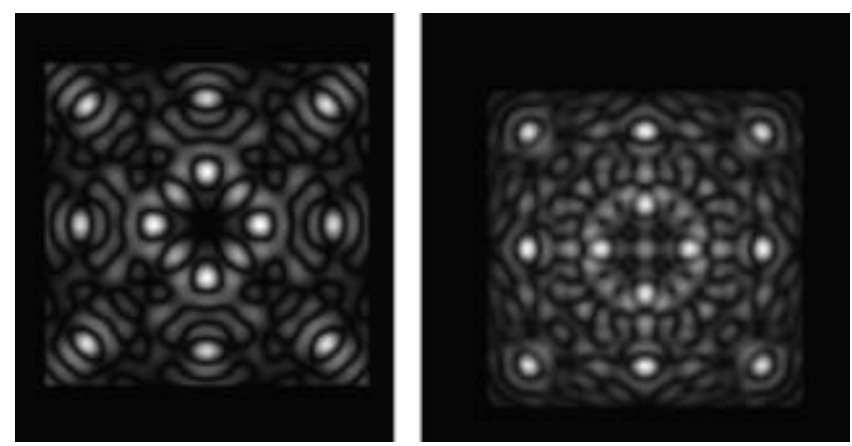

Figure 3. The reconstructed real space atomic arrangement of $\mathrm{Fe}(b c c)$ at two different wavelengths along with the artifacts.

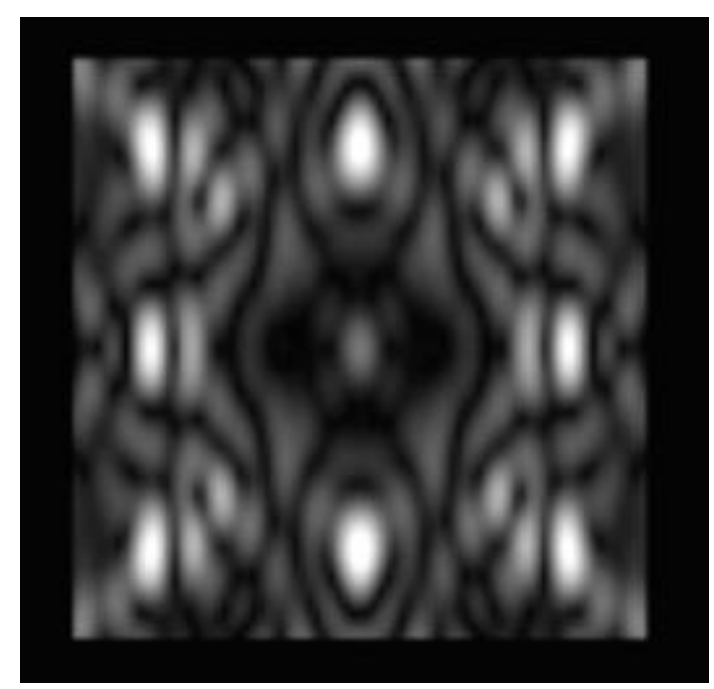

Figure 4. Atomic image of $\mathrm{Fe}(b c c)$ through (010) plane showing the elongation due to incompleteness of the hologram. 


\subsection{Multienergy reconstruction}

In order to remove all the artifacts and go beyond the diffraction limits one should go for a rigorous and more precise formulation evaluating the effects of various terms. This can be achieved through various optimization techniques. In particular we implemented a simple idea to remove the twin image problem as suggested by Barton (1991). The twin image formation in X-ray holography has similar origin as that in the optical holography. To illustrate this point we have simulated a pair of atoms located along the $z$-axis equidistant from the origin. One atom was placed at $(0,0,3)$ and other at $(0,0,-3)$. In figure 6 one can see that twin image of the atom located at $(0,0,-3)$ appears in plane passing through $z=3$. Since many crystals are centro-symmetric and hence twin

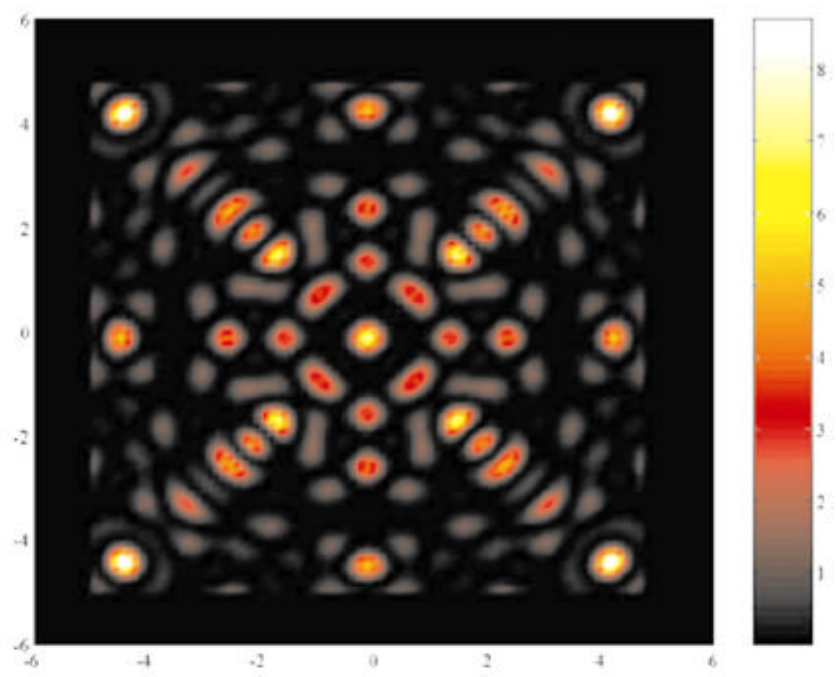

Figure 5. Real space reconstruction of $\mathrm{Cu}(f c c)$ through (001) plane at $k=8^{\circ} \mathrm{A}^{-1}$

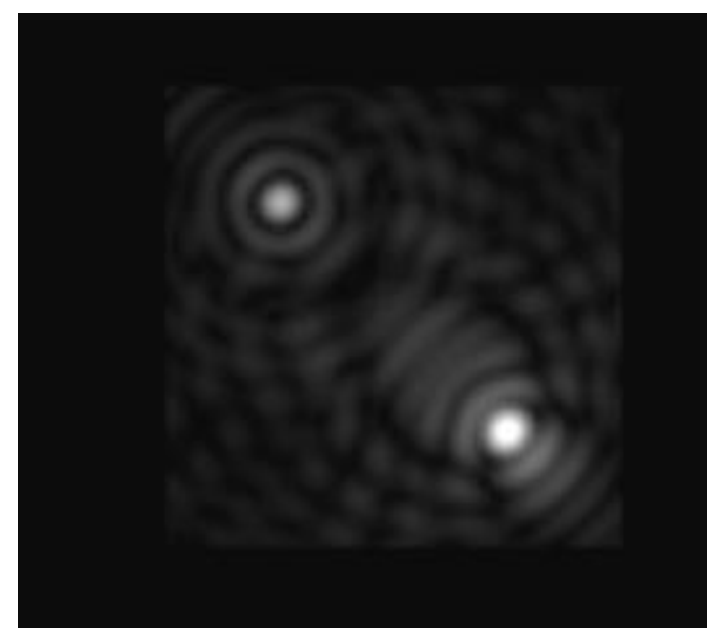

Figure 6. The real and its twin generated for single atom to illustrate the twin image effect. image of one atom is at the same position as the real image of its centro-symmetric counterpart. Since twin and real images have different phases, the complete cancellation of some atomic sites is possible in the reconstructed images. According to Barton's multi-energy algorithm one should collect the hologram at different energies and use them all in the reconstruction process. The energy range is chosen in such a way that the phase terms for twin image oscillate over $2 \pi$ thereby averaging over it, and leads to survival of real image.

This can be achieved using the following reconstruction formula

$$
U(r)=\iint_{s} \chi(\mathbf{k}) \exp i(-\mathbf{k} \cdot \mathbf{r}-k r) k^{2} \mathrm{~d} s \mathrm{~d} k .
$$

The following figures show the multi-energy reconstruction, as done using the above reconstruction formula. It can be clearly seen that many of the artifacts have been removed making the reconstruction much more reliable (figure 7). A simulation was also carried out for $\mathrm{Fe}$ (bcc) with 2000 atoms. Figure 8 shows the reconstruction through (002) plane.

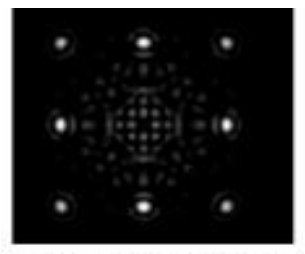

(001)-through emitter

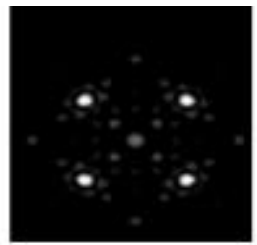

(002)

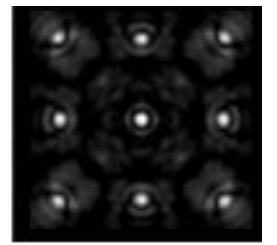

(001)
Figure 7. Reconstruction of $\mathrm{Fe}(b c c)$ using multienergy algorithm for different planes.

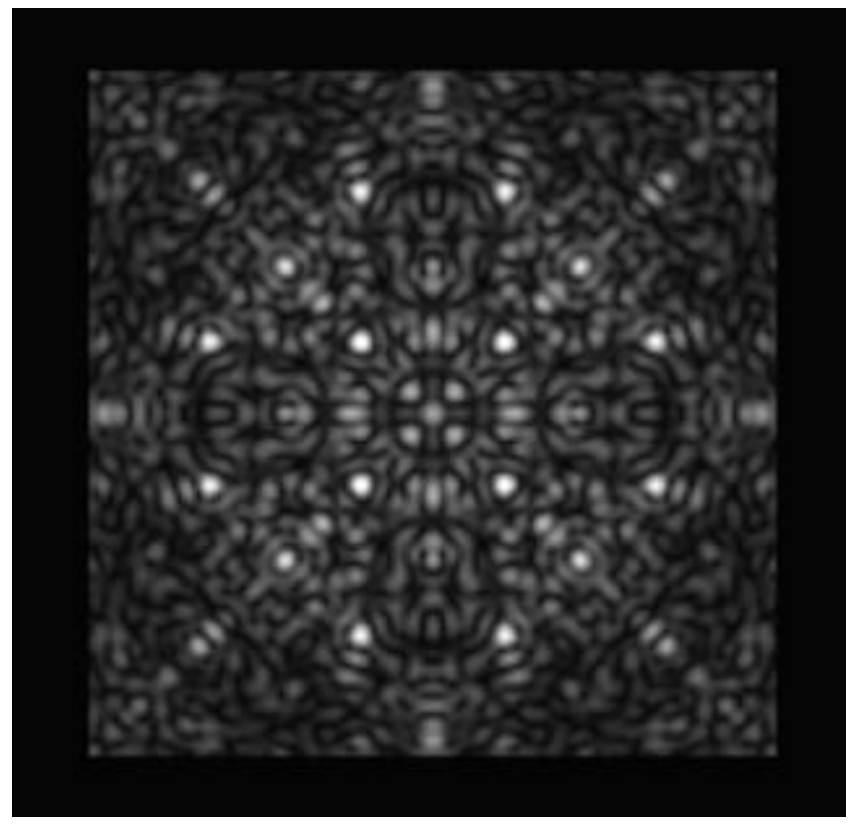

Figure 8. Simulation for 2000 atom for Fe (bcc) through (002) plane showing the second neighbours as well. 
Finally up to now we have produced only $2 \mathrm{D}$ cuts passing through different planes. A naive way of obtaining $3 \mathrm{D}$ image would be to make such $2 \mathrm{D}$ slices and stack them to obtain $3 \mathrm{D}$ images but as we have seen that due to image elongation the image quality would be poor. Hence one needs to go for direct 3D reconstruction using suitable software techniques. We have used the idea of volume rendering or voxel projection to achieve direct $3 \mathrm{D}$ reconstruction. Figure 9 shows the complete 3D reconstruction of $\mathrm{Cu}$ cluster and some of the atomic sites are connected by straight lines so as to avoid any misinterpretation of the reconstructed data.

Finally for algorithm testing we chose a quasicrystal $i-\mathrm{Al}_{72} \mathrm{Pd}_{20} \mathrm{Mn}_{8}$ with the $\mathrm{Pd}$ atoms arranged on the icosahedron in the very first shell. The hologram was generated at $k=8 \mathrm{~A}^{-1}$ in the limited range and then extended to whole sphere using symmetry information. The extended hologram is shown in figure 10 . The five-fold symmetry of the structure is clearly evident from the hologram. It was reconstructed back to get the real space atomic arrangements shown in figure 11 . As can be seen the first shell containing $12 \mathrm{Pd}$ atoms has been clearly reproduced.

\section{Conclusions}

In this paper, we have illustrated some of our simulation work on X-ray holography with some simple structures like iron and copper. These simulations have clearly shown the capability of this technique for imaging the atomic arrangement inside bulk of the material. X-ray holography experiments are being carried out at the synchrotron sources all over the world and the results have proved its capabilities to image not only crystals but also non-periodic systems like quasicrystal, dopants in the

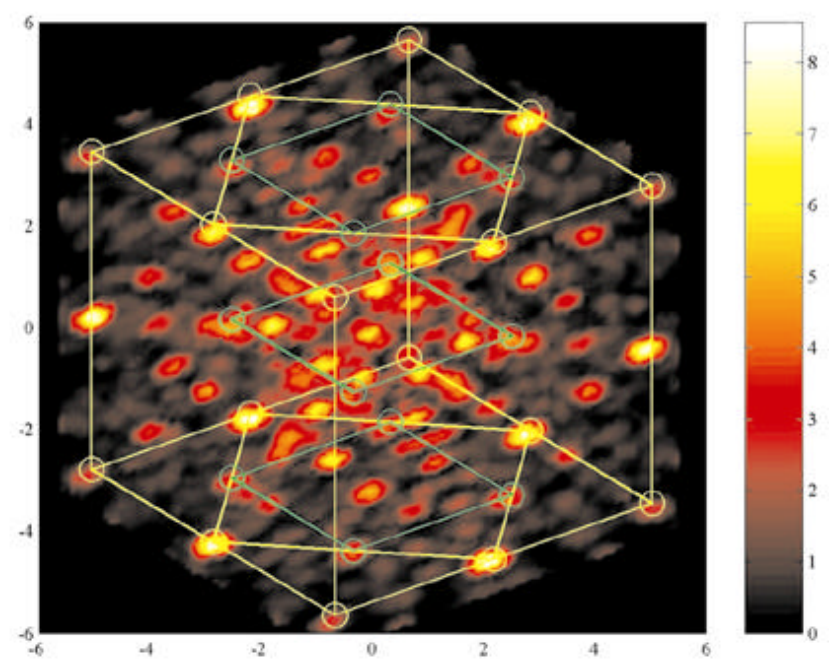

Figure 9. 3D atomic arrangement of $\mathrm{Cu}(f c c)$ using voxel projection for $k=8^{\circ} \mathrm{A}^{-1}$. semiconductor, ultra-thin film etc (Tegze et al 1999; Marchesini et al 2000; Hayashi et al 2001; Takahashi et al 2002). However, in order to exploit its full potential some more developments like detectors with high counting efficiency, new techniques of hologram data collection and $4 \pi$ detectors etc are required. Moreover, to get better and more reliable reconstruction of atomic positions one can look for more sophisticated reconstruction algorithms based on various optimization techniques like maximization of entropy etc. What one could state right

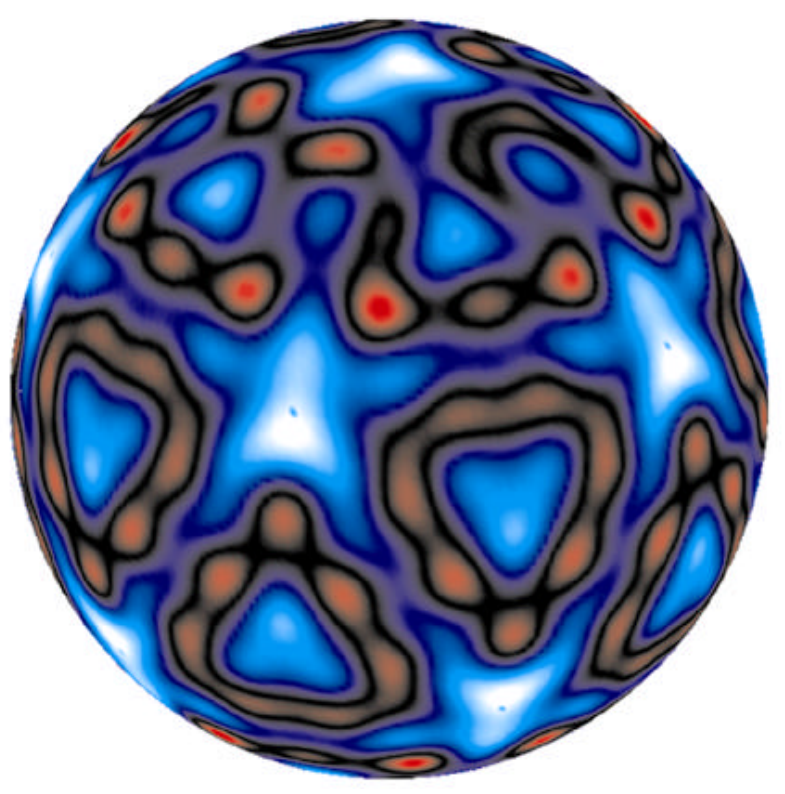

Figure 10. The hologram of $\mathrm{Al}-\mathrm{Pd}-\mathrm{Mn}$ at $E=16 \mathrm{kV}$.

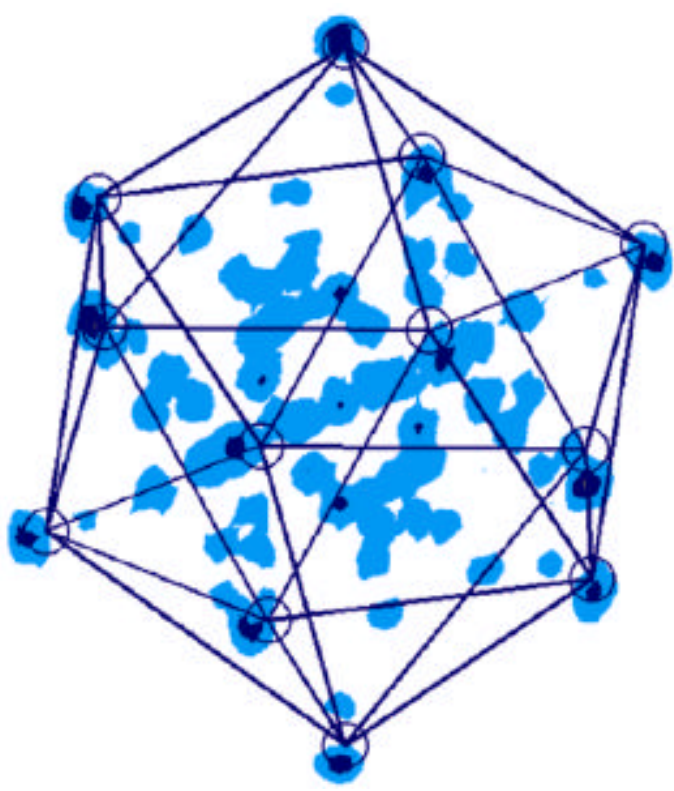

Figure 11. The first shell of $\mathrm{Al}-\mathrm{Pd}-\mathrm{Mn}$, where the $\mathrm{Pd}$ atoms have been connected by lines. 
now is that XFH can be placed between EXAFS which gives high resolution atomic distances with ordered and disordered samples, but lacks complete 3D information, and diffraction on good crystals, which gives information of long range order in periodic system but cannot be applied to disordered systems. The current efforts at the third generation high brilliance synchrotron source to provide the plane wave of sufficient coherence needed in $\mathrm{X}$-ray holography images and reconstruction are being pursued at spring-8 and elsewhere (Hayashi et al 2003; Marchesini and Fadley 2003).

\section{Acknowledgements}

We would like to thank Dr S Marchesini for his valuable help and advice. We are also grateful to Dr R S Rao for his invaluable guidance.

\section{References}

Barton J J 1988 Phys. Rev. Lett. 611356

Barton J J 1991 Phys. Rev. Lett. 673106

Chidambaram R 2002 Proc. of ICQ-8 conf. (to be published)

Gabor D 1948 Nature 61777

Gog T et al 1996 Phys. Rev. Lett. 763132

Hayashi K et al 2001 Phys. Rev. B63 041201R

Hayashi K et al 2003 J. Mater. Sci. 14459

Kashyap Y et al 2003 Poster presented in MRSI symposium,

BARC, Mumbai

Marchesini S and Fadley C S 2003 Phys. Rev. B67 024115

Marchesini S et al 2000 Phys. Rev. Lett. 854723

Modak P et al 2000 Philos. Mag. B80 168

Szoke A 1986 AIP conference proc. (New York: American Institute of Physics) p. 147

Takahashi K et al 2002 Mater. Trans. 431479

Tegze M and Faigel G 1996 Nature 38049

Tegze M et al 1999 Phys. Rev. Lett. 824847 